\title{
APLICACIÓN DE LA TEORÍA DE FRAGMENTACIÓN/ TRANSPORTE SECUENCIAL A LOS DEPÓSITOS DE LAS ERUPCIONES DE 1723 Y 1963-65 DEL VOLCÁN IRAZU, COSTA RICA. CASO DISPERSIÓN NEGATIVA
}

\author{
APPLICATION OF SEQUENTIAL FRAGMENTATION/TRANSPORT THEORY TO \\ DEPOSITS OF 1723 AND 1963-65 ERUPTIONS OF IRAZÚ VOLCANO, \\ COSTA RICA. NEGATIVE DISPERSION CASE
}

\author{
José Brenes \\ Red Ciudadana de Estaciones Meteorológicas, Apartado 290-3015 Costa Rica \\ jbrenes54@gmail.com
}

(Recibido: 29/10/2012 ; aceptado: 11/06/2013)

\begin{abstract}
Sequential fragmentation/transport theory (SFT) was used to analyze tephra deposits of the two main recent explosive eruptions from Irazú volcano, the 1723 and 1963-65 events. The final results provide a new way to analyze fragmentation and transport processes, which fix well with the field observations made by Alvarado (1993). A new way to process fragmentation/transport revealed 4 phi ranges that can be associated to initial volcanic fragmentation. The SFT fragmentation coefficient method is expanded to include all 5 subpopulations, allowing to compare the 1723 and 1963-65 events. SFT interpretation of 1723 eruptions is in good agreement with the volcanological description of such explosive events, whereas SFT points to a more complex 1963-65 eruption, probably due to volcanic recycling and sedimentary reworking as it is observed in the field.

Keywords: Sequential fragmentation/transport, 1723 and 1963-65eruptive units, Irazú volcano, Costa Rica, Volcanic Explosivity Index

RESUMEN: La Teoría de Fragmentación y Transporte Secuencial (SFT) fue aplicada a los depósitos de las dos erupciones explosivas más importantes y recientes del volcán Irazú, los eventos de 1723 y de 1963-65. Los resultados obtenidos proveen una nueva forma de analizar la fragmentación y el transporte, además, coinciden bastante bien con las observaciones de campo realizadas por Alvarado (1993). Este método, aunque fue desarrollado para presentar los datos de transporte, evidenció 4 ámbitos de phi que pueden ser asociados a la fragmentación inicial del magma. El método del coeficiente de fragmentación fue también ampliado para incluir todas las subpoblaciones, permitiendo así comparar físicamente los eventos eruptivos de 1723 y 1963 65. Los resultados muestran que la interpretación de SFT, para los depósitos de 1723, concuerdan bien con las descripciones vulcanológicas de dichos eventos explosivos; mientras que la interpretación para la erupción de 1963-65, resulta más compleja, probablemente debido al reciclaje y retrabajo (re-sedimentación) de las tefras, tal y como se observa en los depósitos eruptivos. Palabras claves: Fragmentación y Transporte Secuencial,, análisis granulométricos, volcán Irazú, Costa Rica.
\end{abstract}




\section{INTRODUCCIÓN}

El volcán Irazú es uno de los volcanes más activos de Costa Rica y de la Cordillera Volcánica Central, registrando varios 5 eventos eruptivos durante las últimas tres centurias: 1723, 1917-20, 1928, 1930, 1933, 1939, 1939-40, y el más reciente en 1963-65. Su relativa cercanía con la infraestructura habitacional, de salud, industria, centros financieros, educativos y agrícolas del país, a solo unas decenas de kilómetros, lo vuelven, sin embargo, un foco de preocupación que debe ser analizado desde todos los ángulos posibles. Por ello, cualquier método que permita obtener información adicional a partir de registros tefroestratigráficos se vuelve de mucha utilidad. En este manuscrito se muestra que la teoría de fragmentación y transporte secuencial (que denominaremos SFT por su nombre en inglés: Sequential Fragmentation/Transport Theory), al aplicarla a las distribuciones granulométricas y estratigráficas incluidas en Alvarado (1993), es un instrumento muy útil para analizar procesos eruptivos. Aquí la aplicaremos a las que se sucedieron en 1723 y 1963-65.

El presente trabajo está dividido en 6 partes: a) una breve descripción de los eventos principales de 1723 y de 1963-65; b) una presentación de los aspectos medulares de la SFT; c) presentación de la metodología utilizada para aplicar la SFT a los datos originales a partir de la granulometría incluida en Alvarado (1993); d) análisis de los datos de 1723 que pone en evidencia una distribución en phi constante a través del tiempo, e incluye una comprobación de la coherencia interna de los mismos; e) la presentación de los resultados de 1963-65 con un breve análisis, debido al menor número de datos y a su mayor complejidad; y f) una descripción de cómo algunos de los procedimientos propuestos en SFT pueden ser ampliados para obtener mayor información.

\section{EVENTOS DE 1723 Y 1963-65}

Por la facilidad de acceso al cráter, el tipo de erupciones, la posibilidad de efectuar mediciones de los efectos que la caída de ceniza tiene en diversas actividades económicas, el evento de 1963-65 marca un hito en el desarrollo de la ciencia vulcanológica costarricense (Alvarado, 1993). Aunado a esto, la posibilidad de poder analizar registros estratigráficos (Fig. 1) nos brinda una muy peculiar oportunidad de poder analizar los dos eventos explosivos principales históricos del Irazú, y a la vez aplicar una metodología nunca antes utilizada en depósitos volcánicos en América Central.

Las erupciones de 1723 dieron comienzo con una oleada piroclástica freática (Fig. 2), representada por una capa delgada $(<10 \mathrm{~cm})$ de ceniza hidrotermalmente alterada, cubierta por $6 \mathrm{~m}$ de tefras basálticas andesíticas, compuesta en su mayoría por lapilli altamente vesicular (22-59\%) y 1-7 vol.\% de fragmentos líticos hidrotermalmente alterados. El aspecto textural de los fragmentos juveniles, las imágenes con el microscopio electrónico de barrido (SEM), unida a descripciones históricas, permite asignarle a esta columna un origen estromboliano. Continúa con 1,2 $\mathrm{m}$ de ceniza gris finamente laminada, y con 1,15 m consistentes en lentes de lapilli, capas de ceniza incluyendo material grueso de bombas y bloques. El pasaje de depósitos cohesivos a no cohesivos es claro y no muestra superficies erosionadas (Alvarado, 1993).

Por su parte, la secuencia de 1963-65 está compuesta por lapilli altamente vesicular con 12$49 \%$ de fenocristales $(9-40 \%$ de plagioclasas y $1-13 \%$ de piroxenos); así como fragmentos provenientes de lavas antiguas y brechas del conducto, que fueron expulsadas en la erupción.

Los resultados de los análisis granulométricos presentados en Alvarado (1993) fueron obtenidos de capas provenientes de una sección de 28 $\mathrm{m}$, localizada en la parte oeste del Cráter Principal (identificados por el código Ira2/*), y de otra sección localizada a unos $300 \mathrm{~m}$ y $80 \mathrm{~m}$ más abajo del cráter principal (identificada como Ira1/*) (Fig. 1) Los histogramas de las muestras están divididos en intervalos de $0,5 \varphi$, lo que nos da una mejor resolución.

Los datos se obtuvieron ampliando los histogramas de las distribuciones granulométricas de 


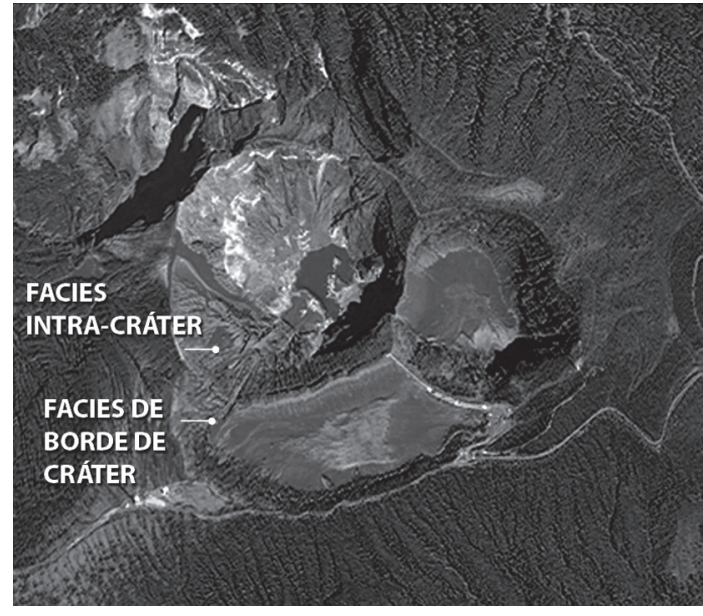

Fig. 1: Posición aproximada de los lugares donde se recolectaron las muestras (para detalles, ver Alvarado, 1993). Tomada de GOOGLE EARTH (2012), en $9^{\circ} 58^{\prime} 50^{\prime \prime} \mathrm{N}, 83^{\circ} 50^{\prime} 53^{\prime}$ 'W.

Alvarado (1993; p. 105, 134 y 135) a 3 veces su tamaño original y midiendo lo más precisamente posible los valores de $\%$ en peso, que fueron asociados al valor menor de $\varphi$, dado que las columnas no se encontraban centradas al medio del intervalo. Aunque no fue posible establecer cuantitativamente el error asociado a este procedimiento, estimamos que no afecta los resultados obtenidos dado que SFT incluye la relación de cada $\varphi$ con la de sus vecinos. Tampoco se afecta la granulometría si se desplazada completamente por $0,25 \varphi$ para centrar las columnas. Todos los resultados de la aplicación de SFT serán identificados con el mismo código de las muestras utilizado por Alvarado (1993).

\section{SOBRE LA TEORÍA SFT}

La granulometría de tefras o depósitos sedimentarios provee información de mucha utilidad sobre los posibles mecanismos de origen asociados: fragmentación, transporte y depositación. En geología para obtener resultados numéricos, es usual aproximar la distribución de los datos a una distribución Gaussiana, lo que puede inducir

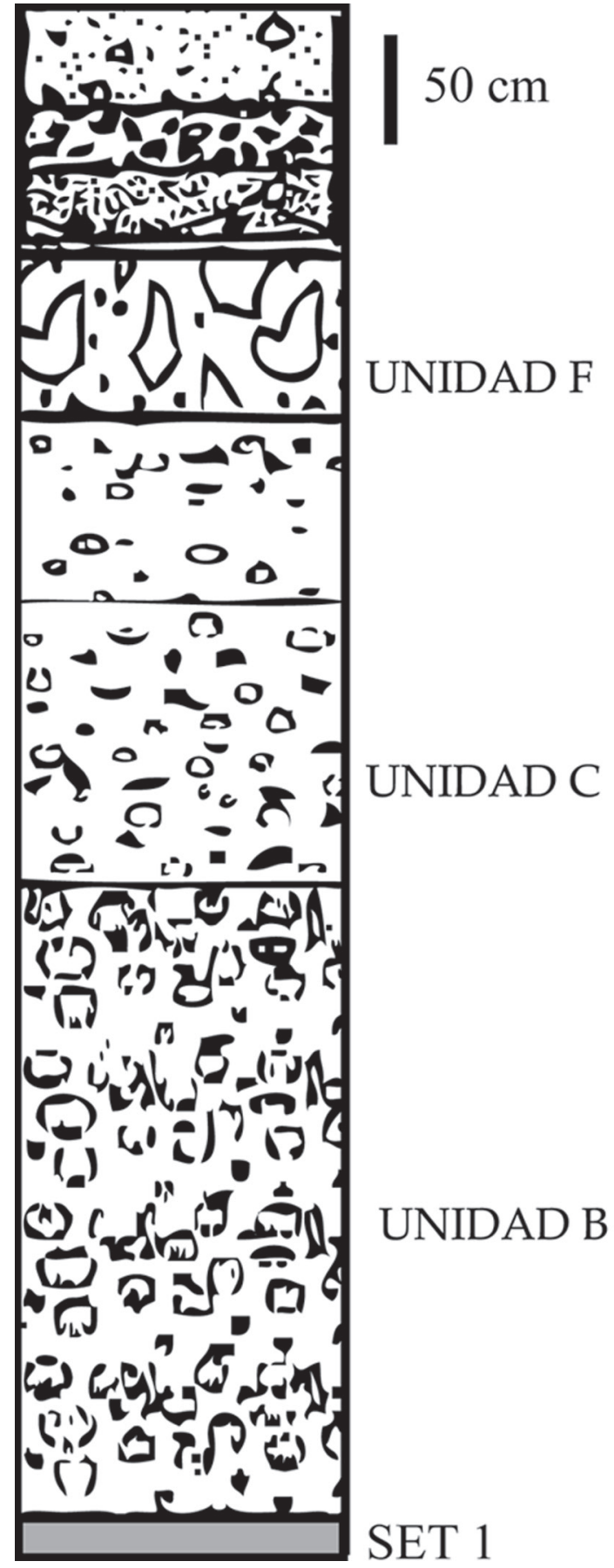

Fig. 2: Estratigrafía simplificada de 1723 (basada en Alvarado, 1993). 
a creer que refleja mecanismos físicos, cuando en realidad solo es una buena aproximación matemática. La distribución de Weibull, por su parte, si tiene un sustento físico como lo derivaron Brown \& Wohletz (1995), en un artículo que además de mostrar lo correcto de considerar el proceso de fragmentación como un proceso fractal, da las bases para la SFT.

La SFT es producto de un análisis general del proceso de fragmentación, hecho por Brown (1989), inicialmente aplicado a nivel astronómico. En un esfuerzo conjunto de Brown con los vulcanólogos K. Wohletz y M. Sheridan, teoría que fue aplicada posteriormente a los depósitos volcánicos (Brown \& Wohletz, 1995: Wohletz et al., 1989), haciendo uso de conceptos físicos para modelar el proceso de fragmentación, así como su posterior elutriación durante el transporte. Las partículas son consideradas esféricas y de densidad constante, para evitar lidiar con las dificultades de morfologías irregulares y variación petrográfica. El punto de partida es que las distribuciones granulométricas reflejan la producción de fragmentos pequeños resultante de la aplicación de procesos mecánicos a fragmentos más grandes, lo que da un carácter fractal a las granulometrías, es decir las vuelve invariantes frente a variaciones de escala.

La SFT propone una curva invariante en la que un parámetro ajustable, denominado dispersión, determina el ancho de la distribución. Si solo se considera la fragmentación, la dispersión se simboliza por $\gamma$, en tanto que se usa $g$ cuando se incluye el transporte. En ambos casos, la dispersión debe ser mayor a -1 , y su valor aumenta conforme la distribución incluye un mayor número de secuencias de fragmentación y/o transporte. Una dispersión cercana a -1 se asocia a una explosión súbita, y se alejará de ese valor límite en casos como ball milling (molienda de bolas)" o bien de transportes complejos como el de saltación. Las granulometrías obtenidas en el campo se consideran como una mezcla de varios procesos de origen y transporte diverso, que se analizan como la suma de varias distribuciones o subpoblaciones, cada una centrada en un valor determinado de phi (conocido como moda) con una dispersión diferente (Wohletz et al., 1989). La precisión de esta descomposición se mide comparando que tanto se acerca la suma de las subpoblaciones, cada una con un peso relativo apropiado conocido como fracción, a la granulometría original (Fig. 2). Teóricamente se pueden asociar intervalos de dispersión $\gamma$ a diversos mecanismos por su relación con la masa $m$.

Así por ejemplo, la nucleación vesicular es directamente proporcional a $\mathrm{m}^{1 / 3}$ (limitación por volumen) y a m ${ }^{2 / 3}$ (limitación por área), lo que lleva a esperar que $\gamma$ variará en el ámbito de $-1 / 3$ a $-2 / 3$ para casos de una fragmentación magmática. Siguiendo los argumentos de Wilson (1972), las partículas pequeñas muestran un comportamiento balístico proporcional a $\mathrm{m}^{-1 / 3}$, en tanto las partículas muy finas muestran un tiempo de suspensión proporcional a $\mathrm{m}^{-2 / 3}$, por lo que los valores de $\mathrm{g}$ para este tipo de transporte se espera estén en el ámbito de $-2 / 3$ a $-1 / 3$. El trasporte por saltación se esperaría que muestre valores de $\mathrm{g}$ en el ámbito $-2 / 3<\mathrm{g}<-1 / 6$ (White, 1979).

La descripción detallada de la física detrás de estos análisis puede ser consultada en Wohletz et al (1989), especialmente la relación que tiene la dispersión $\gamma$ con la dimensión fractal asociada $(\mathrm{D}=-3 \gamma)$. Así mismo, la ecuación (36) de Wohletz et al. (1989), puede ser aplicada para obtener la desviación estándar relacionada con la distribución gaussiana que matemáticamente describiría la distribución.

\section{METODOLOGÍA}

Cada una de las granulometrías obtenidas de Alvarado (1993) fue analizada con el programa SFT (Los Alamos National Laboratory computer code LA-CC 99-29, version 2.19.0168, July 2007, Los Alamos, New Mexico) desarrollado por Wohletz en 1999, basado en las ecuaciones de Wohletz et al. (1989). La idea básica es que cada una de las granulometrías se puede descomponer en varias curvas, a las que se le asociará un mecanismo específico de fragmentación, y una o varias de trasporte (Fig. 3). Los valores discretos de peso porcentual de la distribución granulométrica de cada muestra se transforman en una curva continua, a partir de la 


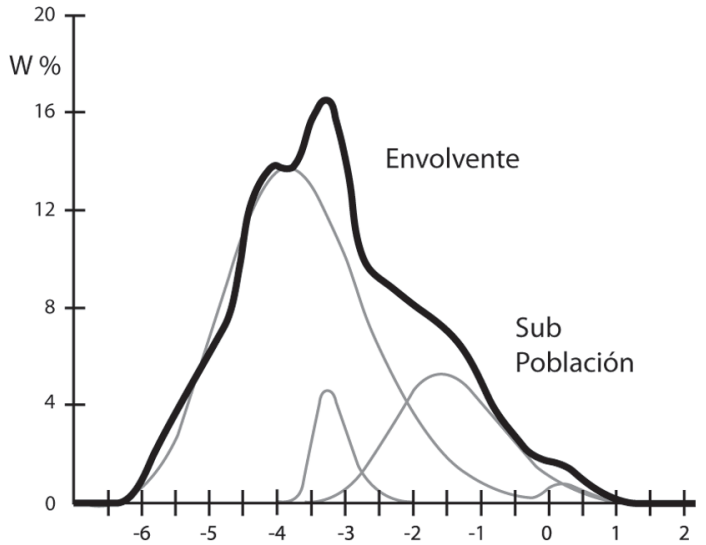

Fig. 3: Ejemplo de granulometría del Irazú deconvolucionada utilizando SFT.

cual el investigador debe identificar, visualmente, las posibles subpoblaciones presentes, procediendo luego a optimizar la escogencia. Cuán correcta sea esa elección, se mide calculando el residuo porcentual entre la envolvente calculada y la distribución original. En nuestro caso, el proceso se repitió hasta lograr un residuo de 4 a $6 \%$. Los resultados finales para ambas erupciones, tanto para fragmentación como fragmentación/transporte, pueden ser consultados en los Apéndices 1 a 4. En algunos casos, las columnas de datos fueron desplazadas para ayudar al lector a identificar patrones. La nomenclatura utilizada para la identificación, y los criterios estratigráficos y eruptivos para agrupar los resultados, es la propuesta en Alvarado (1993), especialmente para la división de las capas estratigráficas denominadas Set I a Set V, y Unidades de A hasta H. Esta manera de obtener información, a partir de la granulometría, usando SFT, puede resultar útil para relacionar los productos derivados de la interacción del agua con el magma o freatomagmáticos (Dellino \& La Volpe, 1996; Zimanowsky et al., 1997; Buttner et al., 1999), o con la tasa de ascenso del magma (Wohletz, 1986; Houghton et al., 1999; Morrisey et al., 2000; Maria \& Carey, 2002). Igualmente, puede ayudar en casos de repetidas transiciones entre actividad magmática (estromboliana) e hidrovolcánica con oleadas piroclásticas húmedas y secas (Sottili et al., 2009; Brand \& Clarke, 2009), o para identificar y reproducir la secuencia de eventos freatomagmáticos (Buttner et al., 2002), y evaluar el potencial de riesgo en áreas activas (Wohletz, 1986; Lorenz, 1986, 1987; Brand \& Clarke, 2009; Sottili et al., 2009).

\section{ANÁLISIS DE LA ERUPCIÓN DE 1723}

Los resultados obtenidos por SFT serán analizados con la ayuda de los valores teóricos esperados, y de los resúmenes experimentales propuestos por Wohletz et al. (1989), quienes previenen que algunos de ellos son algo tentativos; luego serán comparados con los resultados del análisis que se propone en Alvarado (1993). Por no contarse con un método similar a los mínimos cuadrados, y para reducir el grado de subjetividad en el análisis, la comparación se llevará a cabo con la ayuda de procesos subsidiarios, que se irán presentando conforme se requieran

\section{Coherencia interna de los parámetros}

La coherencia del análisis puede ser comprobada definiendo un parámetro de fragmentación $\left(\Phi_{\text {Moda }} / \gamma\right)$ que al graficarse contra la moda de cada población, según lo describe Wohletz et al. (1995), genere una línea recta que pasa cerca del origen con pendiente negativa. Aquellos resultados con dispersión positiva se analizan en un segundo trabajo (Brenes \& Alvarado, 2013). En nuestro caso, aún cuando la composición química del Irazú y el origen multigenético de sus tefras es muy singular (Alvarado et al., 2006), se obtiene una recta con pendiente negativa que pasa cerca del origen, con $\mathrm{R}^{2}>0,9$, como predice la teoría. Procediendo de manera similar a como la presentan Wohletz et al. (1995), al analizar la variación de cada subpoblación con la distancia al cráter, los resultados se agruparon según la subpoblación a que pertenece, obteniéndose para cada una de ellas una recta con pendiente negativa, con $\mathrm{R}^{2}$ generalmente mayor al obtenido cuando se utilizaron todos los resultados en conjunto (Fig. 4). Se interpreta esto como evidencia de que durante la erupción hay procesos constantes, posiblemente 


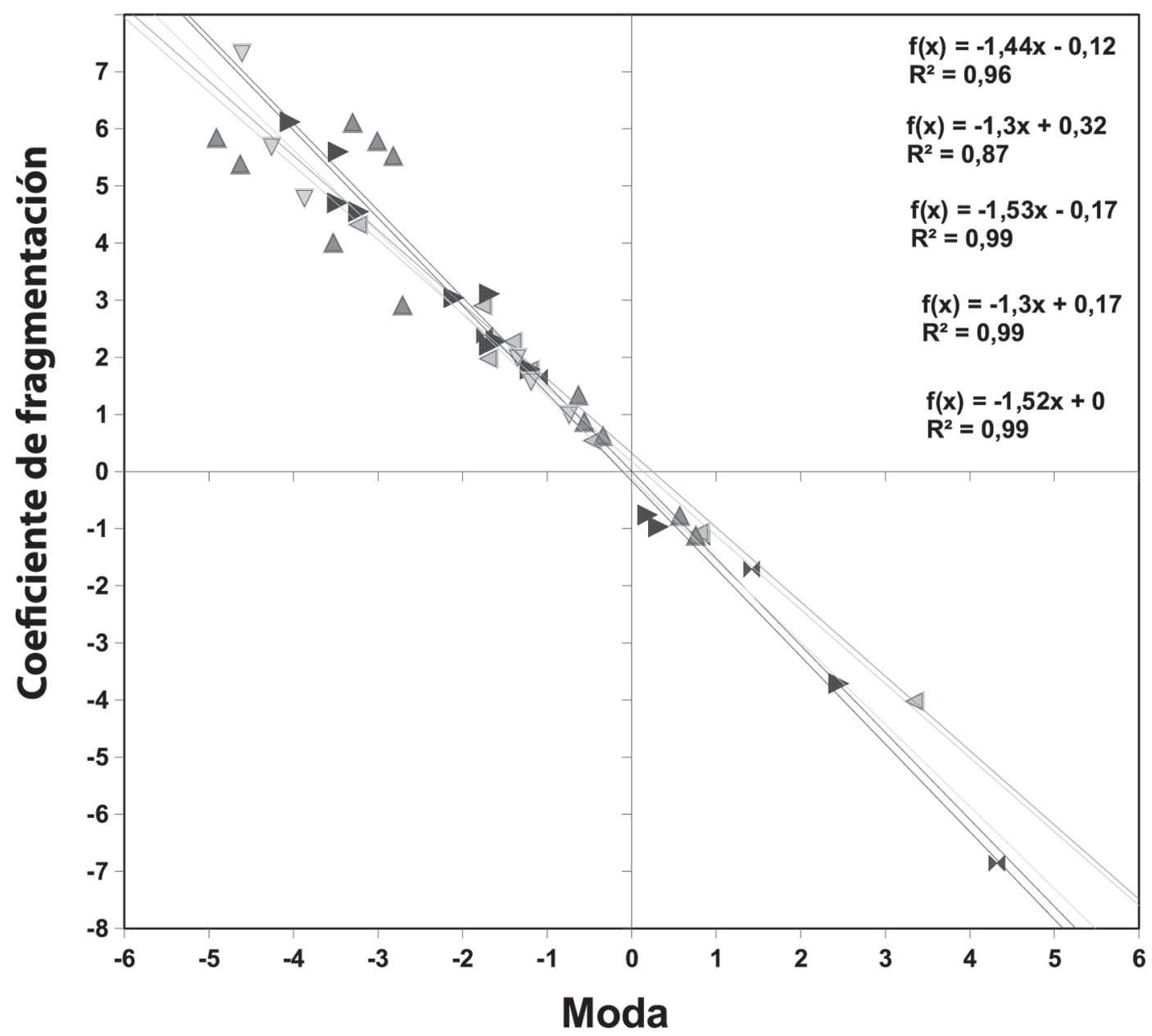

Fig. 4: Evento de 1723, con 15 muestras y un total de 48 puntos. De arriba hacia abajo, las primeras 5 ecuaciones están ordenadas por subpoblación, y la última corresponde a la recta obtenida al incluir todas las subpoblaciones.

establecidos por la longitud del conducto, la composición química de la lava, y la fragmentación, entre otros. Este punto se retomará más adelante al comparar las erupciones de 1723 y 1963-65.

\section{Análisis de la erupción utilizando el modo de fragmentación}

Todas las 15 granulometrías presentadas en Alvarado (1993) fueron analizadas con SFT, utilizando el modo de fragmentación, obteniéndose 64 resultados. De ellos, solo 50 dieron una dispersión negativa y serán utilizados en el análisis. El análisis de los otros 14 resultados, todos con dispersión positiva, se presenta en un segundo trabajo (Brenes \& Alvarado, 2013). Los datos primarios pueden consultarse en el Apéndice 1.

\section{Casos con $\gamma$ negativa}

Wohletz et al. (1989) propusieron un ámbito de $-0,94<\gamma<-0,50$ para ceniza estromboliana 
y de $-0,53<\gamma<+0,47$ para la hidrovolcánica. El traslape en nuestro caso fue resuelto usando la observación de campo de la Ira1/2 (dispersión $\gamma=$ $-0,52$ ) que fuertemente apunta a un origen hidrovolcánico. Por ende, el ámbito $-0,53<\gamma<+0,47$ se asoció exclusivamente con tefra hidrovolcánica. Los valores de $\gamma$ para cada subpoblación, se graficaron (Cuadro 1) con intervalos centrados en $(\mathrm{n}+0,25) \varphi$ у $(\mathrm{n}+0,75) \varphi, \mathrm{n}$ un entero positivo, dado que las granulometrías iniciales, divididas en intervalos de $0,5 \varphi$, no estaban centradas en el intervalo. Se utilizó la etiqueta $\mathrm{H}$ para hidrovolcánico y M para magmático, seguida por su porcentaje relativo. Se ve claramente que, aunque las subpoblaciones Ira1/2 hasta Ira1/9 se encuentran en el ámbito $-4,5 \varphi$ a $-1,5 \varphi$ como se esperaba de la clasificación estratigráfica, las modas se concentran alrededor de tres valores: $-4,25 \varphi$ y $-1,25 \varphi$, asociados a un origen magmático, y la moda de $-3,25 \varphi$ que es producto de una mezcla de actividad magmática (estromboliana) e hidrovolcánica. Este hecho concuerda con la propuesta de Sheridan et al. (1987), de que la aparición de subpoblaciones y desviaciones estándar en los mismos lugares sugiere un origen físico, posiblemente relacionado al tipo de clasto y su densidad (cristales, líticos, pómez). Similarmente, las columnas 0,75 y 1,25 pueden asociarse a un solo mecanismo magmático, que se evidencia en la Ira1/2, y tiempo después en las muestras Ira1/10, Ira1/11, Ira1/12 y en Ira1/19 e Ira1/20, de la Unidad F. Como Sheridan et al. (1987) indican, la existencia y tamaño de ese tipo de subpoblaciones puede heredarse de la fragmentación inicial del

Cuadro 1

Erupción de 1723: Asignación de origen hidrovolcánico $(\mathrm{H})$, o estromboliano $(\mathrm{E})$ a cada subpoblación resultado del análisis por fragmentación, seguido del porcentaje del total de la muestra. Dividido en dos partes dado las diferentes distribuciones de $\varphi$.

Valores de $\varphi \leq-5,25$ no se incluyen por estar muy cerca del límite de detección

\begin{tabular}{|c|c|c|c|c|c|c|c|c|c|c|c|c|c|c|c|c|c|c|}
\hline $\mathrm{Phi} \rightarrow$ & 4,75 & 4,25 & $-3,75$ & $-3,25$ & $-2,75$ & $-1,75$ & $-1,25$ & $-0,75$ & $-0,25$ & 0,25 & 0,75 & 1,25 & 1.75 & 2.25 & 2.75 & 3.25 & 3.75 & 4.25 \\
\hline Ira1/15 & & & & & & & E24 & E 21 & & H55 & & & & & & & & \\
\hline Ira1/14 & & & & & & H 6 & & H55 & & H37 & & & & & & & & \\
\hline Ira1/13 & & & & & & & E41 & & $\begin{array}{l}\mathrm{H} \\
48\end{array}$ & H 11 & & & & & & & & \\
\hline Ira1/12 & & & & H 3 & E 72 & & E 14 & & & & E 10 & & & & & & & \\
\hline Ira1/11 & & & $\mathrm{H} 10$ & H 7 & & $\begin{array}{c}E \\
26\end{array}$ & & & E21 & & & E24 & & & & & & \\
\hline Ira1/10 & & & E24 & E36 & & & E28 & & & & E6 & & & & & & & \\
\hline Ira1/9 & & E36 & & H 7 & E51 & H 7 & & & & & & & & & & & & \\
\hline Ira1/8 & & E 69 & & $\mathrm{H} 21$ & & H 7 & & & & & & & & & & & & \\
\hline Ira1/7 & E23 & & & E49 & & $\begin{array}{c}\mathrm{E} \\
21\end{array}$ & & & & & & & & & & & & \\
\hline Ira1/6 & & E41 & & E35 & & E 6 & & & & & & & & & & & & \\
\hline Ira1/5 & E23 & & & E56 & & $\begin{array}{c}\mathrm{E} \\
17\end{array}$ & & & & & & & & & & & & \\
\hline Ira1/4 & & E 71 & & H6 & & $\begin{array}{l}\mathrm{H} \\
21\end{array}$ & & & & H 1 & & & & & & & & \\
\hline $\operatorname{Ira} 1 / 2$ & & $\mathrm{H} 8$ & & $\mathrm{H} 54$ & & H 4 & & & & & & E34 & & & & & & \\
\hline $\operatorname{Ira} 1 / 20$ & & & & & & & & & & & E49 & & & H9 & & & E33 & E9 \\
\hline Ira/19 & & & & & & & & E8 & & & E49 & & & E18 & & H6 & & E23 \\
\hline
\end{tabular}


magma y la roca aledaña, lo que quizá explique el carácter dual de la columna -3,25 Ira $1 / 13$, Ira $1 / 14$, Ira $1 / 15$ por sus características freatomagmáticas son una unidad diferente.

En la muestra Ira1/12 sobresale el valor de $\gamma=-0,93$ para la moda $-2.71 \varphi$, cercana a la de la columna $-3,25 \varphi$. En Wohletz et al. (1987) se indica que los valores más bajos de $\gamma$ observados en las distribuciones granulométricas de los depósitos del Cráter Elegante (Sonora, México; $\gamma=-0.95$ ) reflejan la distribución inicial. En el caso de los depósitos plinianos de Mount St. Helens, se observa que la posición de las modas iniciales son determinadas por el más bajo valor de $\gamma=-0,94$. Estas predicciones están acordes al origen hidrovolcánico de las muestras de Cráter Elegante, en oposición al origen magmático de las muestras de pómez del Mount St. Helens. Este tipo de consideraciones constituyen un apoyo a la aplicabilidad del SFT al Irazú, pues demuestra que aunque esos depósitos estrombolianos presentan distribuciones asimétricas y son algunas veces irregulares, (Alvarado, 1993, p. 113), la aplicación de SFT revela un patrón coherente.

\section{Análisis de la erupción de 1723 usando la opción fragmentación/transporte}

Las 15 granulometrías fueron re-analizadas para incluir la elutrificación debida al transporte. Aunque Wohletz et al. (1989) proponen unos ámbitos teóricos posibles, éstos no incluyen opciones como forma irregular, variación de densidad, etc., por lo que se prefiere adoptar los ámbitos experimentales cuidadosamente leídos de su gráfico, dejando espacio para ajustes posteriores (Cuadro 2). Procesos por suspensión, en el que la partícula se mueve con movimientos horizontales y verticales entremezclados, saltación y balística, serán también considerados. Los casos de traslape entre diversos ámbitos de la dispersión g serán dilucidados usando los valores de la moda. Los
Cuadro 2

Erupción de 1723: Ámbitos de g usados para asociar los procesos de transporte

\begin{tabular}{lll}
\hline Mecanismo & Teoría & Gráfico \\
\hline Balístico $(\mathrm{B})$ & & $-0,94$ a $-0,65$ \\
Suspensión $(\mathrm{P})$ & & $-0,72$ a 0,05 \\
Saltación $(\mathrm{S})$ & $-2 / 3$ to $-1 / 6$ & $-0,72$ a 0,35 \\
Tracción $(\mathrm{T})$ & $\approx-2 / 3$ & $-0,75$ a $-0,53$ \\
Caída & $-2 / 3$ to $-1 / 3$ & $-0,66$ a $-0,33$ \\
\hline
\end{tabular}

resultados finales pueden consultarse en el Apéndice 2.

Según Sheridan et al. (1987), las partículas de ceniza gruesa a lapilli fino ( 2 a $4 \mathrm{~mm}, \varphi=-1$ a -2) probablemente se mueven por rodamiento en una oleada, mientras que las que tengan un tamaño mediano de $0,12 \mathrm{~mm}(\varphi=3)$, tendrán un tamaño propicio para ser transportadas por saltación, y aquellas con $0,5 \mathrm{~mm},(\varphi=1)$ pueden considerarse que se mueven intermitentemente a saltos y rodando. Por su parte, Wohletz (2001, p. 284) asignó los procesos de transporte de acuerdo a los siguientes ámbitos de $\varphi$ : Balístico para partículas grandes $(\varphi<-4)$, tracción para partículas relativamente grandes $(-4<\varphi<1)$, saltación para partículas relativamente pequeñas $(1<\varphi<4)$, y suspensión para las muy finas $(\varphi>4)$.

Los resultados de la asignación de mecanismos de transporte para el Irazú se presentan en el Cuadro 3. Aquellos casos en que la moda está cerca al límite superior de la granulometría fueron descartados. Los resultados obtenidos por SFT se compararon con los asignados por Alvarado (1993), encontrándose una buena concordancia (Cuadro 4): la muestra Ira1/2 presenta características de una oleada (los porcentajes asociados por SFT a saltación y tracción suman 53\%), mientras que las muestras Ira1/9 e Ira1/10 presentan características balísticas y de oleada, tanto en el campo como por SFT. De manera similar, el transporte balístico deducido 
Cuadro 3

Erupción de 1723: Asignación de origen hidrovolcánico $(\mathrm{H})$, o estromboliano (E) a cada subpoblación resultado del análisis por fragmentación, seguido del porcentaje del total de la muestra. Dividido en dos partes dado las diferentes distribuciones de $\varphi$. Valores de $\varphi \leq-5,25$ no se incluyen por estar muy cerca del límite de detección

\begin{tabular}{|c|c|c|c|c|c|c|c|c|c|c|c|c|c|c|c|c|c|c|c|}
\hline $\mathrm{Phi} \rightarrow$ & $-4,75$ & $-4,25$ & $-3,75$ & $-3,25$ & $-2,75$ & $-1,75$ & $-1,25$ & $-0,75$ & $-0,25$ & 0,25 & 0,75 & 1,25 & 1.75 & 2.25 & 2.75 & 3.25 & 3.75 & 4.25 & 4.75 \\
\hline Ira1/15 & & & & & & & & B31 & PS12 & PS55 & & & & & & & & & \\
\hline Ira1/14 & & & & & & & & B 17 & PS51 & PS32 & & & & & & & & & \\
\hline Ira1/13 & & & & & & & & B49 & PS32 & PS20 & & & & & & & & & \\
\hline Ira1/12 & & & & & B60 & & & B23 & & & & & & & & & & & \\
\hline Ira1/11 & & & S 9 & S 9 & & & BT22 & & B23 & & & B 22 & & & & & & & \\
\hline Ira1/10 & B 12 & & & B44 & & & B31 & & & & & & & & & & & & \\
\hline Ira1/9 & & B29 & & S 5 & B61 & S 4 & & & & & & & & & & & & & \\
\hline Ira1/8 & & B 63 & & S 25 & & & B 9 & & & & & & & & & & & & \\
\hline Ira1/7 & B20 & & & B53 & & & B22 & & & & & & & & & & & & \\
\hline Ira1/6 & & B64 & & S 9 & & & B 13 & & & & & & & & & & & & \\
\hline $\operatorname{Ira} 1 / 5$ & B22 & & & BT45 & & & B27 & & & & & & & & & & & & \\
\hline Ira1/4 & & & & B66 & & & B23 & & & & & & & & & & & & \\
\hline $\operatorname{Ira} 1 / 2$ & & & & TS53 & & & & В 32 & & & & & & & & & & & \\
\hline $\operatorname{Ira} 1 / 20$ & & & & & & & & PS30 & & & B 41 & & S 7 & & & B 41 & & & PS9 \\
\hline $\mathrm{Ira} / 19$ & & & & & & & & B 9 & & & B 41 & & & B 20 & & PS 7 & & B23 & \\
\hline
\end{tabular}

B: balístico, P: suspensión, S: saltación, T: tracción, BT: balístico y/o tracción, PS: suspensión y/o saltación

por SFT para la Unidad F (Ira1/11, Ira1/12), está en acuerdo con los datos de campo, en tanto que las muestras Ira1/13, Ira1/14, Ira1/15 presentan al menos $50 \%$ de componentes de transporte horizontal, tal y como se encuentra en el campo.

\section{Clasificación estratigráfica}

Dado que la dispersión y la moda están relacionadas, se estudiará si la estratigrafía puede derivarse utilizando solo la moda $\varphi$. Un análisis de los resúmenes de fragmentación y transporte (Cuadros 1 y 3 ) revela que un gran porcentaje de los depósitos están en el ámbito de $-4,5 \varphi$ a -1,5 $\varphi$. La suma de los porcentajes en ese ámbito para Ira1/2, Ira1/4, Ira1/5, Ira1/6, Ira1/7 son respectivamente: 66,76 , 73,76 y $70 \%$, sugiriendo fuertemente que pueden colocarse en un solo grupo. El Cuadro 5 sugiere que el ámbito de $-4,5 \varphi$ a $-1,5 \varphi$, que llamaremos $\beta$, es una medida de la presencia o ausencia de las bombas $\left(\mathrm{Md}_{\varphi}=-4,31\right.$ a $\left.-2,91\right)$, una característica distintiva de las observaciones de campo (Alvarado, 1993). Se propone entonces, dividir todo el ámbito de $\varphi$ de cada muestra en 3 segmentos: la suma de las fracciones en el ámbito $-6 \varphi$ a $-4,5 \varphi$, que denominaremos $\alpha$, la parte $\beta$ ya discutida, y la suma de las fracciones de subpoblaciones más finas que $-1,5 \varphi$, que denominaremos $\delta$. Así, cada muestra está representada por la tríada $(\alpha, \beta, \delta)$.

Usando esa tríada, las muestras Ira1/2, Ira1/4, Ira $1 / 11$, e Ira $1 / 12$ pueden agruparse en una clase que llamaremos Clase I-a,b dependiendo de su lugar en la columna, pues comparten $\alpha=0$. Las muestras Ira1/5, e Ira1/7 comparten $\delta=0$, y se agruparán en la Clase II-a,b según la posición en la columna estratigráfica. La muestra Ira $1 / 8$ es única pues $\alpha=\delta=0$, y se le asigna Clase IV. Las muestras Ira1/6, Ira 1/9, e Ira 1/10 comparten $\alpha, \beta$, $\delta \neq 0$ dando lugar a la Clase III-a,b. Las muestras 


\section{Cuadro 4}

Erupción de 1723: comparación de las observaciones de campo (Alvarado, 1993) con resultados obtenidos de SFT

\begin{tabular}{|c|c|c|c|c|c|c|c|}
\hline & Alvarado (1993) & $\mathrm{B}$ & $\mathrm{P}$ & S & $\mathrm{T}$ & $\mathrm{H}$ & $\mathrm{E}$ \\
\hline $\operatorname{Ira} 1 / 20$ & $\mathrm{~B}, \mathrm{~T}, \mathrm{E}$ & 82 & $0-39$ & $0-39$ & & 33 & 67 \\
\hline Ira1/19 & $\mathrm{B}, \mathrm{T}, \mathrm{E}$ & 93 & $0-7$ & $0-7$ & & 6 & 95 \\
\hline Ira1/15 & B, H horizontal & 31 & $0-67$ & $0-67$ & & 55 & 45 \\
\hline Ira1/14 & B, H horizontal & 17 & $0-83$ & $0-83$ & & 98 & \\
\hline Ira1/13 & B, H horizontal & 49 & $0-52$ & $0-52$ & & 59 & 41 \\
\hline Ira1/12 & $\mathrm{B}, \mathrm{E}$ & 83 & & & & 3 & 96 \\
\hline Ira1/11 & $\mathrm{B}, \mathrm{E}$ & $45-67$ & 18 & $0-22$ & & 17 & 71 \\
\hline Ira $1 / 10$ & B, E oleada & 87 & & & & & 94 \\
\hline $\operatorname{Ira} 1 / 9$ & B, E oleada & 90 & & 9 & & 14 & 87 \\
\hline $\operatorname{Ira} 1 / 8$ & B, E caída & 72 & & 25 & & 28 & 69 \\
\hline $\operatorname{Ira1} / 7$ & B, E caída & 95 & & & & & 93 \\
\hline $\operatorname{Ira1} / 6$ & $\mathrm{~B}, \mathrm{E}$ & 77 & & 9 & & & 82 \\
\hline $\operatorname{Ira} 1 / 5$ & $\mathrm{~B}, \mathrm{E}$ & $49-94$ & & & $0-45$ & & 96 \\
\hline $\operatorname{Ira} 1 / 4$ & $\mathrm{~B}, \mathrm{E}$ & 66 & $0-2$ & $0-2$ & & 28 & 71 \\
\hline $\operatorname{Ira} 1 / 2$ & B, T, H oleada & 32 & & $0-53$ & $0-53$ & 66 & 34 \\
\hline
\end{tabular}

B: balística, P: suspensión, S: saltación, T: tracción, H: hidrovolcánica, E: estromboliana.

Dado que varios mecanismos de transporte pueden asociarse simultáneamente a una misma subpoblación, la suma de las columnas B, P, S y T puede ser mayor a 100\%. La suma de las columnas E y H, puede ser menor a 100\% en algunos casos por no incluir valores para $\varphi \leq-5,25$ por estar muy cerca del valor límite de detección.

Ira1/13 hasta Ira1/20 comparten el patrón $\delta=100$, constituyendo la Clase V. La clasificación se resume en las dos últimas columnas del Cuadro 6, en el que también se incluye la clasificación de Alvarado (1993), basada en observaciones de campo.

Hay un acuerdo parcial sugerente de que los valores obtenidos de SFT de hecho tienen información útil en el análisis de los depósitos de ceniza. Las diferencias entre los dos métodos fácilmente se explican porque Alvarado (1993) usó un grupo de propiedades químicas, físicas y morfológicas, en tanto en el presente trabajo solo utilizamos la moda $\varphi$. La Unidad B de Alvarado (1993) puede recuperarse si adicionalmente imponemos la condición ad hoc $\beta>66$, en tanto que la adopción de $\beta>77$ dejaría vacía la Unidad B. Por ende, Alvarado fue capaz de discriminar entre una parte con $\beta<66$ y otra con $77>\beta>66$. Similarmente, la Unidad $\mathrm{C}$ puede recuperarse si únicamente se utiliza la condición $\delta=0$, al igual que la Unidad F se recupera a aplicar solamente el criterio $\alpha=$ 0 . Ofrecemos este análisis como otro argumento a favor de la aplicación del SFT al Irazú.

\section{MECANISMO DE ERUPCIÓN SEGÚN SFT}

Los diferentes análisis que se han presentado hasta aquí, pueden ponerse como una sola unidad al reconstruir, según SFT, la erupción en el tiempo, como se muestra en el Cuadro 3. La columna $-3,25 \varphi$ muestra una alternancia en el tiempo entre transporte balístico y por saltación, lo que sugiere que el evento de 1723 fue lo bastante explosivo como para que los productos se depositaran en el borde del cráter, y permanecieran ahí. La erupción 
Cuadro 5

Parámetros de tamaño de grano representativos de los depósitos de tefra, según Alvarado (1993)

\begin{tabular}{ccccc}
\hline $\begin{array}{c}\text { Parámetro } \\
\text { Inman }\end{array}$ & $\begin{array}{c}\text { Bombas } \\
\text { gruesas }\end{array}$ & $\begin{array}{c}\text { Caida de } \\
\text { lapilli }\end{array}$ & $\begin{array}{c}\text { Oleada } \\
\text { seca }\end{array}$ & $\begin{array}{c}\text { Oleada } \\
\text { húmeda }\end{array}$ \\
\hline $\mathrm{Md}_{\varphi}$ & $-4,31 \mathrm{a}$ & $0,19 \mathrm{a}$ & $0,04 \mathrm{a}$ & $2,29 \mathrm{a}$ \\
$($ Media $)$ & $-2,91$ & 0,3 & 0,56 & 2,73 \\
$\sigma_{\varphi}$ & $1,27 \mathrm{a} 2,0$ & $0,88 \mathrm{a}$ & $0,76 \mathrm{a}$ & $1,82 \mathrm{a}$ \\
(Selección) & & 0,91 & 0,98 & 2,02 \\
\hline
\end{tabular}

de 1723 A.D. del volcán Irazú, a criterio Alvarado (1993, p. 96), fue la más fuerte erupción histórica relacionada con este volcán. Ese depósito inició con una oleada, (Ira1/2), que presenta un $50 \%$ de tracción y saltación. Producto de los movimientos sísmicos registrados, el peso de la gran cantidad de material relativamente suelto lanzado por la erupción, entre otros, se produjeron deslizamientos y transporte por saltación (muestras Ira1/4, Ira1/6, Ira1/8 e Ira1/9). Parte de las muestras Ira1/6 e Ira1/7 pueden asociarse a erupciones que rellenaron el borde. La tracción que se detectó en las muestras Ira1/5 (subpoblación 2) e Ira1/8 (subpoblaciones 2 y 3 ) también sugieren deslizamientos.

Prácticamente, dos tercios de la columna $-4,25 \varphi$ de Ira1/6 e Ira1/8 están asociados a tefra balística, sugiriendo que las erupciones fueron básicamente bombas de $-4 \varphi$ a $-5 \varphi$, lo que no enmascara los mecanismos por saltación de $-3,25 \varphi$. Aunque la columna $-1,75 \varphi$ no refleja ni saltos ni tracción, es un hecho que Ira1/11 si presenta tracción como un posible mecanismo en ese ámbito.

Un cuadro global de la erupción puede obtenerse numerando las muestras en secuencia temporal, calculando el índice de desarrollo, definido como $\mathrm{D}_{\mathrm{i}}=[(1+\gamma) *(5+\mathrm{Moda}) *$ Peso relativo $]$, y graficando cada Di contra el número de muestra (Fig. 5), como se hizo en Tadeucci \& Wohletz (2001). Se usó un límite de 5 en lugar del $\varphi=-6$ que se usa en el trabajo original para controlar los varios casos de $\gamma>0$ alrededor de $\varphi=-6,\left(\mathrm{D}_{\mathrm{i}}<0\right)$. Los ámbitos aproximados de $\varphi$ para cada $\mathrm{D}_{\mathrm{ij}}$ (subpoblación $\mathrm{j}$ de la muestra i) se pueden leer en el Cuadro 7.
Cuadro 6

Comparación de la propuestra estratigráfca (sensu Alvarado , 1993) con la deducida de las tríadas $(\alpha, \beta, \delta)$ descritas en el texto

\begin{tabular}{|c|c|c|c|c|c|c|}
\hline Muestra & Alvarado & $\alpha$ & $\beta$ & $\delta$ & & \\
\hline $\operatorname{Ira} 1 / 20$ & \multirow{7}{*}{ SET III } & \multirow{7}{*}{$\begin{array}{c}\text { Unidad } \\
\mathrm{F}\end{array}$} & 0 & 0 & 100 & \multirow{6}{*}{$\begin{array}{c}\text { Clase } \\
\text { V }\end{array}$} \\
\hline Ira1/19 & & & 0 & 0 & 100 & \\
\hline Ira1/15 & & & 0 & 0 & 100 & \\
\hline Ira1/14 & & & 0 & 0 & 100 & \\
\hline Ira1/13 & & & 0 & 0 & 100 & \\
\hline Ira1/12 & & & 0 & 75 & 24 & \\
\hline Ira1/11 & & & 0 & 23 & 78 & $\begin{array}{c}\text { Clase } \\
\text { I-b }\end{array}$ \\
\hline Ira1/10 & & & 5 & 60 & 34 & \\
\hline Ira1/9 & & D & 26 & 71 & 3 & $\begin{array}{c}\text { Clase } \\
\text { III-b }\end{array}$ \\
\hline Ira1/8 & & & 0 & 100 & 0 & Clase \\
\hline Ira1/7 & SET II & $\begin{array}{c}\text { Unidad } \\
\text { C }\end{array}$ & 29 & 70 & 0 & $\begin{array}{c}\text { IV } \\
\text { Clase } \\
\text { II-b }\end{array}$ \\
\hline Ira1/6 & & & 18 & 76 & 6 & $\begin{array}{c}\text { Clase } \\
\text { III-a }\end{array}$ \\
\hline Ira $1 / 5$ & & $\begin{array}{c}\text { Unidad } \\
\text { B }\end{array}$ & 26 & 73 & 0 & $\begin{array}{c}\text { Clase } \\
\text { II-a }\end{array}$ \\
\hline Ira $1 / 4$ & & & 0 & 76 & 22 & \\
\hline Ira1/2 & SET I & & 0 & 66 & 34 & $\begin{array}{c}\text { Clase } \\
\text { I-a }\end{array}$ \\
\hline
\end{tabular}

La muestra $1\left(\right.$ Ira1/2) presenta $\mathrm{D}_{11} \approx \mathrm{D}_{13} \approx$ 0 , y $\mathrm{D}_{12} \approx \mathrm{D}_{14} \approx 0,5$, indicando un proceso no muy evolucionado, en tanto que las muestras 2 a 10 presentan valores pequeños de $\mathrm{D}_{\mathrm{ij}}$ para cada subpoblación, reflejando un estado estacionario

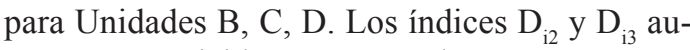
mentan apreciablemente para las muestras 11 a 13 (Ira1/13 a Ira1/15), que muestra un proceso más evolucionado como corresponde al carácter freatomagmático de las muestras. Las últimas muestras presentan $\mathrm{D}_{11} \approx \mathrm{D}_{13} \approx 0$, y $\mathrm{D}_{12} \approx \mathrm{D}_{14} \approx$ 0,75 ; valores similares a la erupción inicial. En resumen, el índice Di es un buen índice para medir la actividad global. 


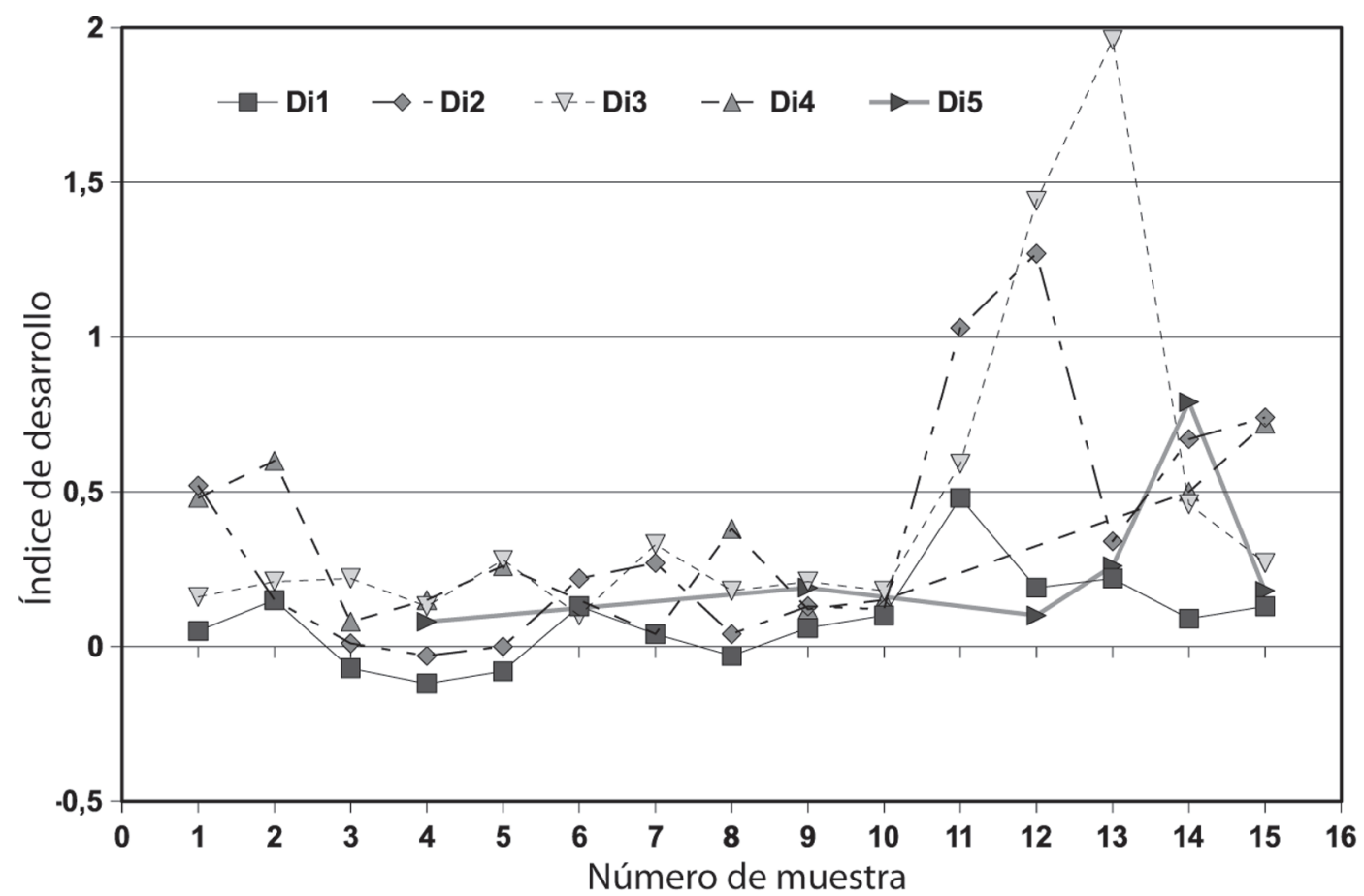

Fig. 5: Evolución de la erupción de 1723, medida con el Índice de Desarrollo (Development Index). Las muestras están numeradas de $1(\operatorname{Ira} 1 / 2)$ a $15(\operatorname{Ira1} / 20)$.

\section{ANÁLISIS Y DISCUSIÓN DE LA ERUPCIÓN DE 1963-65}

Los resultados primarios obtenidos con la opción de fragmentación y de fragmentación/transporte, pueden ser consultados en los Apéndices 3 y 4 , mientras que su análisis se detalla en los cuadros 8 y 9 , respectivamente.

\section{Análisis}

La Fig. 6 muestra que los 27 puntos obtenidos de las 7 muestras, también pueden dividirse en 5 rectas con pendiente negativa que pasan cerca del origen. Las muestras Ira2/3 a Ira 2/7 presentan valores de dispersión relativamente altos, a los que se les puede asociar mecanismos de tracción incluso para finos $(\approx 3,5 \varphi)$ indicativas de una erupción más compleja que la de 1723. En efecto, Alvarado (1993) indica: ...como se infiere de estructuras confiablemente identificadas como oleadas piroclásticas en los depósitos en el borde $(R D)$, sugiriendo que muchos de ellos fueron depositados por turbulentas corrientes que fluyeron de vuelta al cráter.., y ... muchas de ellas [oleadas piroclásticas] posiblemente resultaron de columnas eruptivas colapsadas asociadas con flujos piroclásticos... Las muestras Ira1/30 e Ira1/31 fueron tomadas a unos $300 \mathrm{~m}$ del borde, por lo que son posibles los mecanismos de transporte de tracción, suspensión y saltación evidenciados por SFT. Este reciclaje explica el porqué la erupción de 1963-65 no muestra ningún patrón de $\varphi$ constante. 
Cuadro 7

Ámbitos aproximados de $\varphi$, asociados a las muestras 1 a 10 (Ira1/2 a Ira1/12) y 11 a 15 (Ira1/13 a Ira1/20)

\begin{tabular}{ccc}
\hline Muestra & $1-10$ & $11-15$ \\
\hline Di1 & $-5,0$ a $-3,5$ & $-1,5$ a $-0,5$ \\
Di2 & $-4,5$ a $-3,5$ & $-0,5$ a 0,5 \\
Di3 & $-3,0$ a $-1,5$ & 0,5 a 2,0 \\
Di4 & $-1,5$ a 1,0 & $\approx 3,5$ \\
Di5 & $-1,5$ a 1,5 & $\approx 4,5$ \\
\hline
\end{tabular}

Aunque los resultados para transporte coinciden, las discrepancias en lo referente al origen de algunos depósitos no se pueden resolver. Por ejemplo, un pequeño cambio en el valor mínimo de $\gamma$ asociado al origen hidrovolcánico revierte la predicción de SFT para la muestra Ira1/31 (véase nota en Cuadro 10).

\section{Muestra San José}

Para ser completos, el análisis de la muestra recolectada en San José, a 24 km del cráter, presenta una sóla moda de 2,26Ф, con una dispersión de $-0,55$ correspondiente a transporte por suspensión, típico de depósitos de caída distales.

\section{COMPARACIÓN DE LAS ERUPCIONES DE 1723 Y 1963-65}

El hecho de tener simultáneamente las granulometrías de ambas erupciones, una propuesta de segregar las subpoblaciones al hacer el gráfico de coeficiente de fragmentación versus la moda, y el hecho de que las subpoblaciones se puedan ordenar consecutivamente a partir de la más gruesa sugieren que es posible comparar las dos erupciones comparando las pendientes de las rectas respectivas (Fig. 7). Con el propósito de determinar una incertidumbre aproximada para cada pendiente, se pocedió a calcularlas a partir de tres coeficientes de fragmentación: $\mathrm{C}=(\varphi-0,25) / \gamma, \mathrm{C}=\varphi / \gamma$, y $\mathrm{C}=(\varphi+0,25) / \gamma$, asociados a la misma moda $\varphi$, basados en que el valor de $\gamma$ está asociado a la forma de la granulometría, por lo que no cambiará si la granulometría entera se desplaza por 0,25 en cualquiera de las dos direcciones. El valor de 0,25 corresponde a la mitad del intervalo de $\varphi$ original en que se dividieron las granulometrías. El porcentaje de error de cada pendiente se estimó así en cerca de un $4 \%$. Para el caso específico de la subpoblación 1, la pendiente de la recta para 1723 es mayor que la de 1963-65, lo que implica que la de 1723 fue más explosiva en ese ámbito de $\varphi$. Esto concuerda con lo encontrado por Alvarado

\section{Cuadro 8}

Erupción de 1963-65: Resultados aplicando SFT con solo fragmentación. Muestras Ira1/30 e Ira1/31 fueron recolectadas a unos $300 \mathrm{~m}$ del borde

\begin{tabular}{|c|c|c|c|c|c|c|c|c|c|c|c|c|c|}
\hline $\mathrm{Phi} \rightarrow$ & $-3,75$ & $-2,25$ & $-1,75$ & $-1,25$ & $-0,75$ & $-0,25$ & 0,25 & 0,75 & 1,75 & 2,25 & 3,25 & 3,75 & 4,75 \\
\hline Ira2/7 & H 2 & & & E 37 & & E 48 & & & H 6 & & E 8 & & \\
\hline Ira2/6 & & & & & & & & & E 55 & & & E 45 & \\
\hline $\operatorname{Ira} 2 / 5$ & Н 20 & H 7 & E 66 & & & & & & H 1 & & & & \\
\hline $\operatorname{Ira} 2 / 4$ & & E 23 & & E 25 & & & E 30 & & E4 & & E 16 & & H 2 \\
\hline $\operatorname{Ira} 2 / 3$ & & & & & E 21 & H 12 & & H 23 & H 16 & & Е 27 & & \\
\hline Ira1/30 & & & & & E 14 & & & E 58 & & & E 22 & & H 6 \\
\hline $\operatorname{Ira} 1 / 31$ & & & & & & & H 6 & H 21 & & E 28 & & E 35 & E 10 \\
\hline
\end{tabular}

H: hidrovolcánico, E: estromboliano 


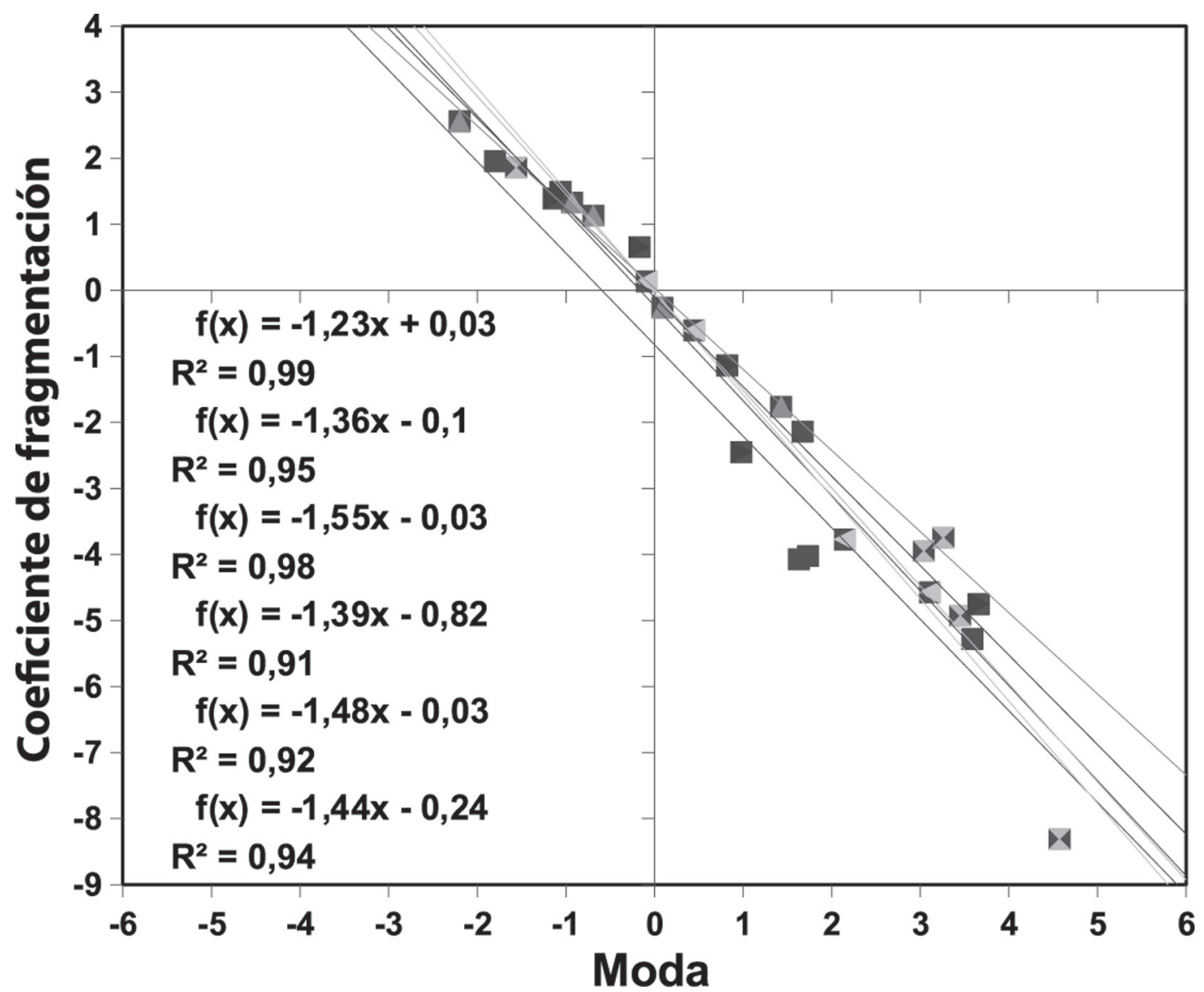

Fig. 6: Evento de 1963-65, con 7 muestras y un total de 27 puntos. Las ecuaciones de las rectas están ordenadas por subpoblación, de 1 a 5 , de arriba abajo. La última ecuación incluye todos los puntos.

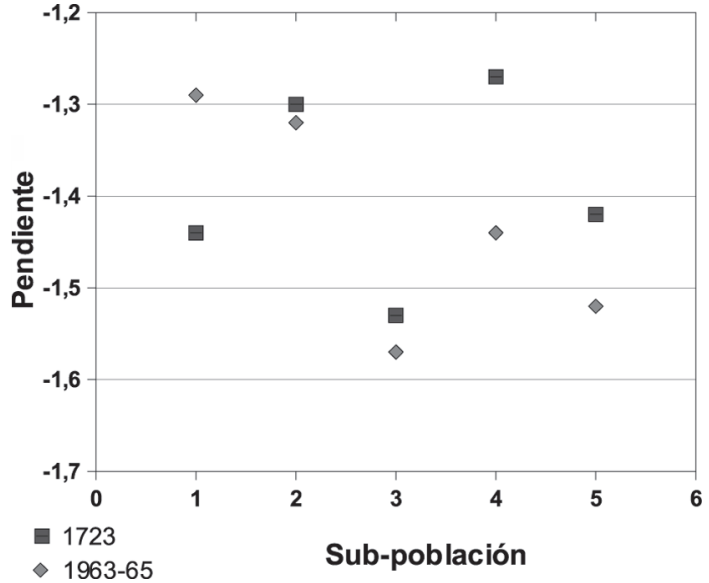

Fig. 7: Gráfico de las pendientes para los eventos 1723 y $1963-$ 65 , según subpoblación. El error de cada pendiente fue estimado en $4 \%$ como caso extremo.
(1993) de que la erupción de 1723 causó un incremento de 50 a $100 \mathrm{~m}$ del borde del cráter en unos pocos días. Las otras 4 pendientes son experimentalmente iguales.

\section{CONCLUSIONES}

La teoría de la fragmentación y transporte secuencial (SFT) por primera vez se aplica a los depósitos de ceniza de un volcán de América Central, encontrándose que da resultados confiables, y que permite analizar con mucho detalle los procesos involucrados en la erupción. Su aplicación a la erupción de 1723 permitió analizarla desde otra perspectiva, al ordenar cronológicamente los valores de dispersión por moda, revelando un patrón 
Cuadro 9

Erupción de 1963-65: resumen de los procesos de transporte según SFT

\begin{tabular}{|c|c|c|c|c|c|c|c|c|c|c|c|c|}
\hline $\mathrm{Phi} \rightarrow$ & $-3,75$ & $-2,25$ & $-1,25$ & $-0,75$ & $-0,25$ & 0,25 & 0,75 & 1,75 & 2,25 & 3,25 & 3,75 & 4,75 \\
\hline Ira2/7 & B 9 & & B 18 & & BT 56 & & & B 15 & & BTS 3 & & \\
\hline Ira2/6 & & & & & & & & B 51 & & & B 49 & \\
\hline Ira2/5 & PS 30 & & B 55 & & & & & PS 4 & & & & \\
\hline Ira2/4 & & B 21 & В 26 & & & BT 32 & & $? ?$ & & BT 16 & & \\
\hline $\mathrm{Ira} 2 / 3$ & & & & BT 6 & PS 15 & & S 19 & BT 27 & & B 23 & & \\
\hline Ira $1 / 30$ & & & & PS 9 & & & В 62 & & & В 24 & & PS 6 \\
\hline Ira1/31 & & & & & & TPS 8 & TPS 27 & & PS 15 & & B 46 & TPS 4 \\
\hline
\end{tabular}

B: balístico, BT: balístico y/o tracción, BTS: balístico y/o tracción y/o saltación, PS: suspensión y/o saltación, TPS: tracción y/o suspensión y/o saltación

Cuadro 10

Erupción de 1963-65: comparación de las observaciones de Alvarado (1993) con resultados de SFT.

\begin{tabular}{cccccccc}
\hline & Alvarado (1993) & B & P & S & T & H & E \\
\hline Ira2/7 & Horizontal > Vertical, H & $42-100$ & & $0-3$ & $3-59$ & 8 & 91 \\
Ira2/6 & Rodamiento, Saltación, H & 100 & & & & 0 & 100 \\
Ira2/5 & Balístico, E & 47 & & & 48 & 34 & 66 \\
Ira2/4 & Rodamiento, Saltos, H & $47-95$ & & & $0-48$ & 2 & 98 \\
Ira2/3 & Rodamiento, Saltos, H & $23-56$ & $0-15$ & $19-34$ & $0-33$ & 51 & 48 \\
Ira1/30 & Tracción, H & 86 & $0-15$ & $0-15$ & & 6 & 94 \\
Ira1/31 ver nota & Horizontal $>>$ vertical, H & 46 & $0-54$ & $0-54$ & $0-39$ & 27 & 73 \\
Ira1/31 ver nota & Horizontal $>>$ vertical, H & 46 & $0-54$ & $0-54$ & $0-39$ & 65 & 35 \\
\hline
\end{tabular}

Nota: El pequeño cambio del límite inferior (de $-0,53<\gamma$ a $-0,57<\gamma$ ), causa un gran cambio en la asignación del origen volcánico

subyacente en el que varios valores característicos de $\varphi$ se repiten a través del evento. La inclusión de la variable transporte reveló otro patrón que a su vez sugirió un posible mecanismo de erupción, derivado todo de la aplicación de SFT. La carencia de un patrón definido en el caso del evento 1963-65 está acorde con el proceso de reciclado observado y derivado de la interpretación de campo, sugiriendo el colapso de la columna como un mecanismo dominante de erupción. El análisis por transporte, usando procesos reconocidos de tracción y saltación, logró discriminar entre los depósitos Intra-Cráter, y los del borde. Además, el método propuesto por Wohletz et al. (1989), de graficar el coeficiente de fragmentación versus la moda, fue ampliado al segregar las rectas según la subpoblación, lográndose así nueva información valiosa que permitió comparar la explosividad de cada erupción. No se llevó a cabo ningún análisis de cómo las subpoblaciones varían con la distancia, por no tenerse las granulometrías apropiadas.

Todo lo anterior sugiere que si se recolectan muestras durante el evento eruptivo, sería posible ir llevando a cabo este tipo de análisis en forma sincrónica, recabando así información útil para la gestión de la emergencia. 


\section{AGRADECIMIENTOS}

Al Dr. Guillermo Alvarado Induni por su interés en estudiar los volcanes de Costa Rica, lo que queda en evidencia al revisar exhaustivamente y mejorar apreciablemente las 6 versiones de este trabajo en solo 2 meses, para asegurar que el punto de vista físico estuviera incluido en este número especial. Al Dr. Kenneth Wohletz, uno de los gestores de la SFT, por haber revisado la penúltima versión. A Wendy Pérez y Andrea Borgia por haber sugerido cambios muy apropiados que, no solo mejoraron el trabajo, sino que lo hicieron más amigable a los que tienen conocimientos geológicos.

\section{REFERENCIAS}

ALVARADO, G. E., 1993. Volcanology and Petrology of Irazú Volcano, Costa Rica.261 págs. Univ. Kiel, Alemania [Tesis Ph.D.].

ALVARADO, G. E., CARR, M. J., TURRIN, B., SWISHER, C., SCHMINCKE, H-U. \& HUDNHUT, K., 2006: Recent volcanic history of Irazú volcano, Costa Rica: Alternation and mixing of two magma batches, and pervasive mixing.- Geol. Soc. Amer. Special Paper, 412: 259-276.

BRAND, B.D. \& CLAKE, A.B., 2009: The architecture, eruptive history, and evolution of the Table Rock Complex, Oregon: from a Surtseyan to an energetic maar eruption.- J. Volcanol. Geotherm. Res. 180: 203-224.

BROWN, W.K., 1989: A theory of sequential fragmentation and its astronomical applications.- J. Astrophys. Astr. 10: 89-112

BROWN, W. \& WOHLETZ, K., 1995: Derivation of the Weibull distribution based on physical principles and its connection to the Rossin-Rammler and lognormal distributions.- J. Appl. Phys. 78: 2758-2763.
BRENES， J. \& ALVARADO, G.E., 2013: Aplicación de la teoría de fragmentación/ transporte secuencial a los depósitos de las erupciones de 1723 y 1963-65 del volcán Irazú, Costa Rica. Caso de dispersión positiva y modelo fractal.- Rev. Geol. Amer. Central, 48: 48: 87-98.

BUTTNER, R., DELLINO, P. \& ZIMANOWSKI, B., 1999: Identifying magma-water interaction from the surface features of ash particles.- Nature, 401: 688-690.

BUTTNER, R.P., DELLINO, P., LA VOLPE, L, LORENZ, V. \& ZIMANOWSKI, B., 2002: Thermohydraulic explosions in phreatomagmatic eruptions as evidenced by the comparison between pyroclasts and products from Molten Fuel Coolant Interaction experiments.- J. Geophys. Res., 107: 2277, DOI:10.1029/2001JB000511.

DELLINO, P. \& LA VOLPE, L., 1996: Image processing analysis in reconstructing fragmentation and transportation mechanisms of pyroclastic deposits: The case of Monte Pilato-Rocche Rosse eruptions, Lipari (Aeolian Islands, Italy).- J. Volcanol. Geotherm. Res. 71: 13-19.

HOUGHON, B.F., WILSON, C.J. \& SMITH, I.E., 1999: Shallow-seated controls on styles of explosive basaltic volcanism: a case study from New Zealand.- J. Volcanol. Geotherm. Res. 91: 97-120.

LORENZ, V., 1986: On the growth of maars and diatremes and its relevance to the formation of tuff-rings.- Bull. Volcanol. 48: 265-274.

LORENZ, V., 1987: Phreatomagmatism and its relevance.- Chemical Geology, 62: 149-156.

MARIA, A. \& CAREY, S., 2002: Using fractal analysis to quantitatively characterize the shapes of volcanic particles.- J. Geophys. Res. 107(B11): 2283, DOI:10.1029/2001JB000822, 2002 
MORRISEY, M., ZIMANOWSKI, B., WOHLETZ, K. H. \& BÜTTNER, R., 2000: Phreatomag matic fragmentation.- En: SIGURDSSON, H. (ed.): Encyclopedia of Volcanoes.- Academic Press, San Francisco: 431-445.

ORSI, G., GALLO, G., HEIKEN, G., WOHLETZ, K., YU, E. \& BONANI, G. 1992: A comprehensive study of pumice formation and dispersal: the Creatio Tephra of Ischia (Italy).- J. Volcanol. Geotherm. Res. 53: 329-354

SHERIDAN, M.F., WOHLETZ, K.H. \& DEHN, J., 1987: Discrimination of grain-size subPoblacións in pyroclastic deposits.Geology, 15: 367-370

SOTTILI, G., TADEUCCI, J., PALLADINO, D.M., GAETA, M., SCRALATO, P., VENTURA, G. 2009: Sub-surface dynamics and eruptive styles of maars in the Colli Albani Volcanic District, Central Italy.- J. Volcanol. Geotherm. Res. 180 (24): $189-202$

TADEUCCI, J. \& WOHLETZ, K.H., 2001: Temporal evolution of the Minoan eruption (Santorini, Greece), as recorded by its Plinian fall deposit and interlayered ash flow beds.J. Volcanol. Geotherm. R. 109: 299-317.

WALKER, G.P. \& CROASDALE, R., 1971: Characteristics of some basaltic pyroclastics.- Bull. Volcanol. 35: 303-317

WHITE, B.R., 1979: Soil transport by winds on Mars.- J. Geophys. Res. 84: 4643-4651
WILSON, L., 1972: Explosive volcanic eruptions, II. The atmospheric trajectories of pyroclasts.- Gephys. J. R. Astron. Soc. 30: 381-392.

WOHLETZ, K.H, 1984: Mechanisms of hydrovolcanic pyroclast formation: Grainsize, scanning electron microscopy, and experimental studies.- J. Volcanol. Geotherm. Res. 17: 31-63.

WOHLETZ, K.H. 1986: Explosive magmawater interaction: thermodynamics, explosion mechanisms and field studies.- Bull. Volcanol. 48: 245-264.

WOHLETZ, K.H., 2001: Modeling physical processes of explosive volcanic eruptions: 247-312.- En: FREUNDT, A. \& ROSI, M. (eds.): From magma to tephra.- Elsevier Science Publications, Amsterdam.

WOHLETZ, K.H., SHERIDAN, M.F. \& BROWN, K. 1989: Particle size distribution and the sequential fragmentation/ transport theory applied to volcanic ash.- J. Geophys. Res. 94: 15,703-15,721

WOHLETZ, K. G., ORSI \&. DE VITA, S., 1995: Eruptive mechanisms of the Neapolitan Yellow Tuff interpreted from stratigraphic, chemical, and granulometric data.- J. Volcanol. Geotherm. Res. 67: 263-290

ZIMANOWSKI, B., BÜTTNER, R., LORENZ, V. \& HÄFELE, G., 1997: Fragmentation of basaltic melt in the course of explosive volcanism.- J. Geophys. Res. 102(B1): 803-814. 
Apéndice 1

Erupción de 1723: resultados de SFT con el modo de solo fragmentación, detallados por subpoblación

\begin{tabular}{|c|c|c|c|c|c|c|c|}
\hline Ira $1 / 2$ & & Población1 & Población2 & Población3 & Población4 & & \\
\hline Moda & & $\neg-4,35$ & $-3,01$ & $-1,76$ & 1,14 & & \\
\hline Dispersión & & 0,02 & $-0,52$ & 0,25 & $-0,77$ & & \\
\hline Fracción & & 0,08 & 0,54 & 0,04 & 0,34 & & \\
\hline Ira1/4 & & Población1 & Población2 & Población3 & Población4 & & \\
\hline Moda & & $\neg-3,87$ & $-3,27$ & $-1,60$ & 0,20 & & \\
\hline Dispersión & & $-0,81$ & 0,49 & $-0,70$ & 0,23 & & \\
\hline Fracción & & 0,71 & 0,06 & 0,21 & 0,01 & & \\
\hline Ira1/5 & Población1 & Población2 & & Población3 & Población4 & & \\
\hline Moda & $-6,67$ & $-4,63$ & & $-3,48$ & $-1,70$ & & \\
\hline Dispersión & 0,31 & $-0,86$ & & $-0,74$ & $-0,86$ & & \\
\hline Fracción & 0,03 & 0,23 & & 0,56 & 0,17 & & \\
\hline Ira $1 / 6$ & Población1 & Población2 & Población3 & Población4 & Población5 & & \\
\hline Moda & $-6,20$ & $-5,27$ & $-4,04$ & $-3,24$ & $-1,09$ & & \\
\hline Dispersión & 0,23 & 0,09 & $-0,66$ & $-0,75$ & $-0,66$ & & \\
\hline Fracción & 0,08 & 0,10 & 0,41 & 0,35 & 0,06 & & \\
\hline Ira1/7 & Población1 & Población2 & & Población3 & Población4 & & \\
\hline Moda & $-6,20$ & $-4,73$ & & $-3,47$ & $-1,77$ & & \\
\hline Dispersión & 0,15 & $-0,76$ & & $-0,62$ & $-0,61$ & & \\
\hline Fracción & 0,06 & 0,23 & & 0,49 & 0,21 & & \\
\hline Ira $1 / 8$ & Población1 & Población2 & Población3 & & & & \\
\hline Moda & $-4,26$ & $-2,82$ & $-1,68$ & & & & \\
\hline Dispersión & $-0,75$ & $-0,51$ & $-0,54$ & & & & \\
\hline Fracción & 0,69 & 0,21 & 0,07 & & & & \\
\hline Ira1/9 & Población1 & Población2 & Población3 & Población4 & & & \\
\hline Moda & $-4,05$ & $-3,18$ & $-2,65$ & $-2,11$ & & & \\
\hline Dispersión & $-0,85$ & $-0,05$ & $-0,87$ & 0,02 & & & \\
\hline Fracción & 0,36 & 0,07 & 0,51 & 0,07 & & & \\
\hline Ira1/10 & Población1 & Población2 & Población3 & Población4 & Población5 & & \\
\hline Moda & $-5,62$ & $-3,53$ & $-3,23$ & $-1,41$ & 0,83 & & \\
\hline Dispersión & 0,01 & $-0,88$ & $-0,71$ & $-0,62$ & $-0,72$ & & \\
\hline Fracción & 0,05 & 0,24 & 0,36 & 0,28 & 0,06 & & \\
\hline Ira1/11 & & Población1 & Población2 & Población3 & Población4 & Población5 & Población6 \\
\hline Moda & & $-3,87$ & $-3,13$ & $-1,68$ & $-0,47$ & 1,42 & 2,78 \\
\hline Dispersión & & $-0,45$ & 0,01 & $-0,76$ & $-0,87$ & $-0,83$ & $-0,57$ \\
\hline Fracción & & 0,10 & 0,07 & 0,26 & 0,21 & 0,24 & 0,13 \\
\hline
\end{tabular}


Apéndice 1

Erupción de 1723: resultados de SFT con el modo de solo fragmentación, detallados por subpoblación (continuación)

\begin{tabular}{|c|c|c|c|c|c|c|}
\hline Ira1/12 & & & Población1 & Población2 & Población3 & Población4 \\
\hline Moda & & & $-3,30$ & $-2,71$ & $-1,2$ & 0,80 \\
\hline Dispersión & & & 0,93 & $-0,93$ & $-0,67$ & $-0,75$ \\
\hline Fracción & & & 0,03 & 0,72 & 0,14 & 0,10 \\
\hline Ira1/13 & Población1 & Población2 & Población3 & & & \\
\hline Moda & $-1,35$ & $-0,34$ & 0,32 & & & \\
\hline Dispersión & $-0,68$ & $-0,54$ & 0,01 & & & \\
\hline Fracción & 0,41 & 0,48 & 0,11 & & & \\
\hline Ira1/14 & Población1 & Población2 & Población3 & & & \\
\hline Moda & $-1,85$ & $-0,63$ & 0,19 & & & \\
\hline Dispersión & 0,01 & $-0,47$ & $-0,25$ & & & \\
\hline Fracción & 0,06 & 0,55 & 0,37 & & & \\
\hline Ira1/15 & Población1 & Población2 & Población3 & & & \\
\hline Moda & $-1,19$ & $-0,56$ & 0,32 & & & \\
\hline Dispersión & $-0,76$ & $-0,64$ & $-0,33$ & & & \\
\hline Fracción & 0,24 & 0,21 & 0,55 & & & \\
\hline Ira1/19 & Población1 & Población2 & Población3 & Población 4 & Población 5 & \\
\hline Moda & $-0,74$ & 0,57 & 2,45 & 3,24 & 4,32 & \\
\hline Dispersión & $-0,75$ & $-0,74$ & $-0,66$ & 0,02 & $-0,63$ & \\
\hline Fracción & 0,08 & 0,46 & 0,18 & 0,06 & 0,23 & \\
\hline $\operatorname{Ira} 1 / 20$ & Población1 & Población2 & Población3 & Población 4 & & \\
\hline Moda & & 0,84 & 2,20 & 3,51 & 4,75 & \\
\hline Dispersión & $-0,87$ & $-0,57$ & $-0,78$ & $-0,62$ & & \\
\hline Fracción & 0,49 & 0,09 & 0,33 & 0,09 & & \\
\hline
\end{tabular}


Apéndice 2

Erupción de 1723: resultados de SFT con el modo de fragmentación/transporte, detallados por subpoblación

\begin{tabular}{|c|c|c|c|c|c|c|c|}
\hline $\operatorname{Ira} 1 / 2$ & & Población 1 & Población 2 & Población3 & Población 4 & & \\
\hline Moda & & $\neg-4,36$ & $-3,00$ & $-1,76$ & $-1,02$ & & \\
\hline Dispersión & & 0,02 & $-0,63$ & 0,01 & $-0,86$ & & \\
\hline Fracción & & 0,08 & 0,53 & 0,07 & 0,32 & & \\
\hline $\operatorname{Ira} 1 / 4$ & & Población 1 & Población 2 & Población 3 & Población 4 & & \\
\hline Moda & & $\neg-3,92$ & $-3,24$ & $-1,66$ & 0,09 & & \\
\hline Dispersión & & $-0,87$ & 0,37 & $-0,78$ & $-0,44$ & & \\
\hline Fracción & & 0,66 & 0,08 & 0,23 & 0,02 & & \\
\hline Ira $1 / 5$ & Población 1 & Población 2 & Población 3 & Población 4 & & & \\
\hline Moda & $-6,59$ & $-4,67$ & $-3,45$ & $-1,95$ & & & \\
\hline Dispersión & 0,01 & $-0,76$ & $-0,73$ & $-0,90$ & & & \\
\hline Fracción & 0,33 & 0,22 & 0,45 & 0,27 & & & \\
\hline Ira $1 / 6$ & Población 1 & Población 2 & Población 3 & Población 4 & Población 5 & & \\
\hline Moda & $-6,20$ & $-5,32$ & $-3,93$ & $-3,16$ & $-1,69$ & & \\
\hline Dispersión & 0,31 & 0,74 & $-0,82$ & $-0,46$ & $-0,86$ & & \\
\hline Fracción & 0,08 & 0,06 & 0,64 & 0,09 & 0,13 & & \\
\hline Ira1/7 & Población 1 & Población 2 & Población 3 & & Población 4 & & \\
\hline Moda & $-6,24$ & $-4,91$ & $-3,47$ & & $-1,74$ & & \\
\hline Dispersión & 0,63 & $-0,84$ & $-0,75$ & & $-0,73$ & & \\
\hline Fracción & 0,05 & 0,20 & 0,53 & & 0,22 & & \\
\hline $\operatorname{Ira} 1 / 8$ & Población 1 & & Población 2 & Población 3 & & & \\
\hline Moda & $-4,30$ & & $-2,86$ & $-1,70$ & & & \\
\hline Dispersión & $-0,81$ & & $-0,63$ & $-0,67$ & & & \\
\hline Fracción & 0,63 & & 0,25 & 0,09 & & & \\
\hline Ira1/9 & Población 1 & Población 2 & Población 3 & Población 4 & & & \\
\hline Moda & $-4,36$ & $-3,28$ & $-2,55$ & $-2,09$ & & & \\
\hline Dispersión & $-0,85$ & 0,47 & $-0,90$ & 0,02 & & & \\
\hline Fracción & 0,29 & 0,05 & 0,61 & 0,04 & & & \\
\hline Ira1/10 & Población 1 & Población 2 & Población 3 & Población 4 & & Población 5 & \\
\hline Moda & $-5,69$ & $-4,55$ & $-3,19$ & $-1,44$ & & 0,73 & \\
\hline Dispersión & 0,41 & $-0,86$ & $-0,80$ & $-0,73$ & & $-0,88$ & \\
\hline Fracción & 0,04 & 0,12 & 0,44 & 0,31 & & 0,09 & \\
\hline Ira1/11 & & Población1 & Población 2 & Población 3 & Población4 & Población5 & Población6 \\
\hline Moda & & $-3,91$ & $-3,11$ & $-1,82$ & $-0,44$ & 1,39 & 2,82 \\
\hline Dispersión & & $-0,47$ & 0,02 & $-0,75$ & $-0,86$ & $-0,86$ & $-0,72$ \\
\hline Fracción & & 0,09 & 0,09 & 0,22 & 0,23 & 0,22 & 0,16 \\
\hline
\end{tabular}


Apéndice 2

Erupción de 1723: resultados de SFT con el modo de fragmentación/transporte, detallados por subpoblación (continuación)

\begin{tabular}{|c|c|c|c|c|c|c|}
\hline Ira1/12 & & & Población 1 & Población 2 & Población3 & Población4 \\
\hline Moda & & & $-3,29$ & $-2,99$ & $-1,17$ & 0,80 \\
\hline Dispersión & & & 0,99 & $-0,95$ & $-0,82$ & $-0,83$ \\
\hline Fracción & & & 0,03 & 0,60 & 0,23 & 0,12 \\
\hline Ira1/13 & Población 1 & Población 2 & Población 3 & & & \\
\hline Moda & $-1,18$ & $-0,39$ & 0,34 & & & \\
\hline Dispersión & $-0,81$ & $-0,52$ & $-0,33$ & & & \\
\hline Fracción & 0,49 & 0,32 & 0,20 & & & \\
\hline Ira1/14 & Población 1 & Población 2 & Población 3 & & & \\
\hline Moda & $-1,24$ & $-0,53$ & 0,22 & & & \\
\hline Dispersión & $-0,88$ & $-0,63$ & $-0,29$ & & & \\
\hline Fracción & 0,17 & 0,51 & 0,32 & & & \\
\hline Ira1/15 & Población 1 & Población 2 & Población 3 & & & \\
\hline Moda & $-1,06$ & $-0,26$ & 0,33 & & & \\
\hline Dispersión & $-0,81$ & $-0,73$ & $-0,46$ & & & \\
\hline Fracción & 0,31 & 0,12 & 0,55 & & & \\
\hline Ira1/19 & Población 1 & Población 2 & Población 3 & Población 4 & Población 5 & \\
\hline Moda & $-0,63$ & 0,59 & 2,38 & 3,29 & 4,37 & \\
\hline Dispersión & $-0,81$ & $-0,80$ & $-0,75$ & 0,02 & $-0,74$ & \\
\hline Fracción & 0,09 & 0,41 & 0,20 & 0,07 & 0,23 & \\
\hline $\operatorname{Ira} 1 / 20$ & Población 1 & Población 2 & Población 3 & Población 4 & Población 5 & \\
\hline Moda & $-0,85$ & 0,75 & 1,89 & 3,34 & 4,69 & \\
\hline Dispersión & 0,01 & $-0,79$ & $-0,29$ & $-0,84$ & $-0,66$ & \\
\hline Fracción & 0,03 & 0,41 & 0,07 & 0,41 & 0,09 & \\
\hline
\end{tabular}


Apéndice 3

Erupción de 1963-65: resultados de SFT con el modo de solo fragmentación, detallados por subpoblación

\begin{tabular}{|c|c|c|c|c|c|c|}
\hline $\operatorname{Ira} 2 / 3$ & Población 1 & Población 2 & Población 3 & Población 4 & Población 5 & \\
\hline Modo & $-0,93$ & $-0,17$ & 0,86 & 1,63 & 3,26 & \\
\hline Dispersión & $-0,70$ & $-0,26$ & 0,02 & $-0,40$ & $-0,87$ & \\
\hline Fracción & 0,21 & 0,12 & 0,23 & 0,16 & 0,27 & \\
\hline $\operatorname{Ira} 2 / 4$ & Población 1 & Población 2 & Población 3 & Población 4 & Población 5 & Población 6 \\
\hline Modo & $-2,20$ & $-1,06$ & 0,45 & 1,67 & 3,04 & 4,43 \\
\hline Dispersión & $-0,86$ & $-0,71$ & $-0,74$ & $-0,78$ & $-0,77$ & $-0,19$ \\
\hline Fracción & 0,23 & 0,25 & 0,30 & 0,04 & 0,16 & 0,02 \\
\hline $\operatorname{Ira} 2 / 5$ & Población 1 & Población 2 & Población 3 & Población 4 & Población5 & \\
\hline Modo & $-4,67$ & $-3,73$ & $-2,76$ & $-1,51$ & 1,74 & \\
\hline Dispersión & 0,27 & $-0,06$ & $0,32^{\text {oo }}$ & $-0,91$ & 0,01 & \\
\hline Fracción & 0,06 & 0,20 & 0,07 & 0,66 & 0,01 & \\
\hline $\operatorname{Ira} 2 / 6$ & Población 1 & Población 2 & & & & \\
\hline Modo & 1,43 & 3,66 & & & & \\
\hline Dispersión & $-0,81$ & $-0,77$ & & & & \\
\hline Fracción & 0,55 & 0,45 & & & & \\
\hline Ira2/7 & Población 1 & Población 2 & Población 3 & Población 4 & Población5 & \\
\hline Modo & $-3,84$ & $-1,14$ & $-0,09$ & 1,73 & 3,45 & \\
\hline Dispersión & $-0,27$ & $-0,82$ & $-0,67$ & $-0,43$ & $-0,70$ & \\
\hline Fracción & 0,02 & 0,37 & 0,48 & 0,06 & 0,08 & \\
\hline $\operatorname{Ira} 1 / 30$ & Población 1 & Población 2 & Población 3 & Población 4 & & \\
\hline Modo & $-0,69$ & 0,82 & 3,11 & 4,67 & & \\
\hline Dispersión & $-0,61$ & $-0,72$ & $-0,68$ & $-0,31$ & & \\
\hline Fracción & 0,14 & 0,58 & 0,22 & 0,06 & & \\
\hline Ira1/31 & Población 1 & Población 2 & Población 3 & Población 4 & Población 5 & \\
\hline Modo & 0,09 & 0,98 & 2,15 & 3,59 & 4,57 & \\
\hline Dispersión & $-0,34$ & $-0,40$ & $-0,57$ & $-0,68$ & $-0,55$ & \\
\hline Fracción & 0,06 & 0,21 & 0,28 & 0,35 & 0,10 & \\
\hline
\end{tabular}


Apéndice 4

Erupción de 1963-65: resultados de SFT con el modo de fragmentación/transporte, detallados por subpoblación

\begin{tabular}{|c|c|c|c|c|c|c|}
\hline $\operatorname{Ira} 2 / 3$ & Población1 & Población 2 & Población 3 & Población 4 & Población 5 & \\
\hline Modo & $-1,11$ & $-0,21$ & 0,82 & 1,53 & 3,57 & \\
\hline Dispersión & $-0,70$ & $-0,31$ & 0,02 & $-0,73$ & $-0,92$ & \\
\hline Fracción & 0,16 & 0,15 & 0,19 & 0,27 & 0,23 & \\
\hline Ira2/4 & Población1 & Población 2 & Población 3 & Población 4 & Población 5 & Población 6 \\
\hline Modo & $-2,29$ & $-0,99$ & 0,51 & 1,72 & 2,95 & 4,83 \\
\hline Dispersión & $-0,9$ & $-0,95$ & $-0,79$ & 0,43 & $-0,75$ & 0,79 \\
\hline Fracción & 0,21 & 0,26 & 0,32 & 0,03 & 0,16 & 0,02 \\
\hline $\operatorname{Ira} 2 / 5$ & Población1 & Población 2 & Población 3 & Población 4 & & \\
\hline Modo & $-3,73$ & $-2,71$ & $-1,33$ & 1,41 & & \\
\hline Dispersión & $-0,51$ & 0,42 & $-0,93$ & $-0,77$ & & \\
\hline Fracción & 0,30 & 0,09 & 0,55 & 0,04 & & \\
\hline $\operatorname{Ira} 2 / 6$ & Población 1 & Población 2 & & & & \\
\hline Modo & 1,39 & 3,65 & & & & \\
\hline Dispersión & $-0,88$ & $-0,87$ & & & & \\
\hline Fracción & 0,51 & 0,49 & & & & \\
\hline Ira2/7 & Población 1 & Población 2 & Población 3 & Población 4 & Población 5 & \\
\hline Modo & $-2,23$ & $-1,41$ & $-0,14$ & 1,87 & 3,83 & \\
\hline Dispersión & $-0,94$ & $-0,80$ & $-0,79$ & $-0,92$ & $-0,68$ & \\
\hline Fracción & 0,09 & 0,18 & 0,56 & 0,15 & 0,03 & \\
\hline $\operatorname{Ira} 1 / 30$ & Población 1 & Población 2 & Población 3 & Población 4 & & \\
\hline Modo & $-0,92$ & 0,75 & 3,09 & 4,69 & & \\
\hline Dispersión & $-0,5$ & $-0,83$ & $-0,81$ & $-0,43$ & & \\
\hline Fracción & 0,09 & 0,62 & 0,24 & 0,06 & & \\
\hline Ira1/31 & Población 1 & Población 2 & Población 3 & Población 4 & Población 5 & \\
\hline Modo & 0,3 & 1,20 & 2,20 & 3,64 & 4,67 & \\
\hline Dispersión & $-0,68$ & $-0,71$ & $-0,59$ & $-0,85$ & $-0,62$ & \\
\hline Fracción & 0,08 & 0,27 & 0,15 & 0,46 & 0,04 & \\
\hline
\end{tabular}


